\title{
DISTRIBUCIÓN Y ABUNDANCIA DEL PEZ LEÓN PTEROIS VOLITANS EN LA ZONA COSTERA DE USO TURÍSTICO DE HOLGUÍN, CUBA
}

\author{
Distribution and abundance of the lionfish Pterois volitans \\ in tourist coastal zone of Holguín, Cuba
}

\author{
Enrique Reynaldo ${ }^{1}$, Antonio Vega ${ }^{2}$, Alejandro Fernández ${ }^{2}$, José Cruz ${ }^{2}$, \\ Elier Córdova ${ }^{2}$ y Pedro Cruz $^{2}$
}

\begin{abstract}
${ }^{1}$ Instituto de Ciencias del Mar (ICIMAR). Ave. 1ra. No. 18406 e/ 184 y 186, Flores, Playa, La Habana. ereynaldodelacruz@gmail.com.

${ }^{2}$ Centro de Investigaciones y Servicios Ambientales de Holguín.

Calle 18 s/n e/ 1ra. y Maceo. Reparto. El Llano, Holguín, 80100, Cuba.

vega@cisat.cu, ale@cisat.cu,jose@cisat.cu, elier@cisat.cu,pedro@cisat.cu.
\end{abstract}

\section{RESUMEN}

En Cuba, a partir del 2007, se detectó la presencia de Pterois volitans, comúnmente llamado Pez León, especie invasora causante de grandes daños a comunidades ictiológicas autóctonas en diferentes regiones del mundo. Este trabajo se propuso determinar la distribución y abundancia del Pez León en diferentes sitios de muestreo en la zona costera de uso turístico de Holguín. El estudio se realizó en ocho campañas de muestreo durante el período abril-noviembre de 2016. Se seleccionó un total de siete sitios de monitoreo distribuidos en dos municipios de la provincia Holguín (Rafael Freyre y Banes). Se determinó la abundancia promediando los valores de cada recorrido lineal (individuo por cada $100 \mathrm{~m}^{2}$ ). Se determinó la talla y el peso por estación de muestreo con una cinta métrica y una balanza digital. La comparación múltiple de medias se realizó por PERMANOVA con 10000 permutaciones empleando la distancia euclidiana. Se registró un total de 365 individuos. El sitio con mayor densidad fue Playa Blanca, con $12.42 \mathrm{ind} / 100 \mathrm{~m}^{2} \mathrm{y}$ el de menor abundancia playa Don Lino, con $3.66 \mathrm{ind} / 100 \mathrm{~m}^{2}$, detectando solamente diferencia estadística entre este sitio con el resto de los sitios monitoreados. El mes con mayor densidad en el año de estudio fue abril, con $13.14 \mathrm{ind} / 100 \mathrm{~m}^{2}$, registrándose diferencias estadísticas significativas en cuanto a la abundancia entre este y los meses de mayo $\left(6.42 \mathrm{ind} / 100 \mathrm{~m}^{2}\right)$, julio $\left(7.28 \mathrm{ind} / 100 \mathrm{~m}^{2}\right)$ y agosto $\left(6.71 \mathrm{ind} / 100 \mathrm{~m}^{2}\right)$. La comparación de la abundancia poblacional entre años del Pez León, registrada en el 2016 con años anteriores demuestra diferencia estadística entre los años $2013(p<0.001)$ y $2012(p<0.01)$.

Palabras clave: distribución, abundancia, Pterois volitans, Holguín.

\section{ABSTRACT}

In Cuba, as of 2007, the invasion of Pterois volitans was detected, commonly called lionfish, an invasive species that causes great damage to indigenous ichthyological communities in different regions of the world. This work was proposed to determine the distribution and abundance of lionfish in different sampling sites in the coastal zone of tourist use of Holguín. The study was conducted in eight sampling campaigns during the period from April to November 2016. A total of seven monitoring sites were selected distributed in two municipalities of Holguín province (Rafael Freyre and Banes). The abundance was determined by averaging the values of each linear travel (individual per $100 \mathrm{~m}^{2}$ ). Size and weight were determined by sampling station with a measuring tape and digital balance. The multiple means comparison was performed by PERMANOVA with 10000 permutations using the Euclidean distance. Were registered 365 individuals, including all sampling sites. 
The site with the highest density was Playa Blanca, with $12.42 \mathrm{ind} / 100 \mathrm{~m}^{2}$ and the lowest abundance Don Lino beach, with 3.66 ind $/ 100 \mathrm{~m}^{2}$, detecting only statistical difference between this site and the rest of the monitored sites. The month with the highest density in the year of study was April with $13.14 \mathrm{ind} / 100 \mathrm{~m}^{2}$, registering significant statistical differences in terms of abundance between this and the months of May $\left(6.42 \mathrm{ind} / 100 \mathrm{~m}^{2}\right)$, July $\left(7.28 \mathrm{ind} / 100 \mathrm{~m}^{2}\right)$ and August $\left(6.71 \mathrm{ind} / 100 \mathrm{~m}^{2}\right)$. The comparison of the population abundance among years of the lionfish recorded in 2016 with previous years shows statistical differences between the years $2013(p<0.001)$ and $2012(p<0.01)$.

Keywords: distribution, abundance, Pterois volitans, Holguín.

\section{INTRODUCCIÓN}

Con el nombre de Pez León se conoce a varias especies del género Pterois, que pertenecen a la familia Scorpaenidae. Se caracterizan por sus vistosas aletas en forma de pluma o abanico, de colores que varían de marrón a rojizo, con franjas rayas verticales blancas a crema, las cuales le proporcionan camuflaje. Presentan veneno en la base de las espinas dorsales, pélvicas y anales (Green et al., 2012; Morris et al., 2011). Las zonas tropicales del Indo-Pacífico son el ámbito de distribución natural del Pez León, no obstante, dos especies (Pterois miles y P. volitans) han invadido el Atlántico Occidental y mar Caribe, desde la Florida, golfo de México, Antillas Mayores y Menores, Centro América hasta la costa norte de Sur América, por lo que su invasión es una de las más rápidas y extensas documentadas para peces marinos (Morris y Akins, 2009).

En Cuba se registró a partir del año 2007 y se ha dispersado rápidamente por toda la isla (Caballero et al., 2008; Chevalier et al., 2008). Por su gran capacidad de diseminación y reproducción es una especie muy difícil de eliminar y controlar, significando una amenaza para la ecología de los arrecifes (Alcolado, 2008). Esta especie es un carnívoro muy voraz, que puede causar afectaciones en las comunidades de arrecife de coral por depredación directa de peces nativos e invertebrados y compitiendo con sus posibles depredadores (Albins y Hixon, 2008). En su dieta se incluyen peces pequeños y juveniles de importancia económica como los meros. El Pez León tiene la posibilidad de diezmar poblaciones de especies importantes como los herbívoros claves, controladores de las algas. Además, consume invertebrados como crustáceos y moluscos y es notablemente rápido en adaptarse a consumir nuevas presas (Albins y Hixon, 2011).

Según Morris y Whitfield (2009) se ha observado en el Caribe una disminución en muchas actividades turísticas y en numerosos lugares se han puesto señales de alarma para evitar envenenamiento con las espinas del Pez León. Estas medidas afectan la economía de las regiones turísticas donde se ha detectado la presencia del Pez León. Estos impactos negativos sobre las comunidades nativas, combinados con otros factores como el cambio climático, la contaminación y la sobrepesca, pudieran traer afectaciones aún mayores y provocar grandes pérdidas de la biodiversidad marina en los arrecifes de coral (Albins y Lyons, 2012).

\section{OBJETIVO}

-Determinar la distribución espacial y abundancia del Pez León en la zona costera de uso turístico de la provincia de Holguín. 


\section{MATERIALES Y MÉTODOS}

Área de estudio. Esta investigación se realizó en una costa sin plataforma de origen tectónico, abarcando la zona turística del litoral desde Playa Blanca (X-587 200; Y-270 700 ) hasta Playa Guardalavaca (X-605 450; Y-275 410), con una longitud aproximada de $35 \mathrm{~km}$ de línea de costa, sin contar los contornos de las bahías de Vita y Naranjo (Fig. 1). La profundidad de trabajo osciló entre 5-45 $\mathrm{m}$ de profundidad.

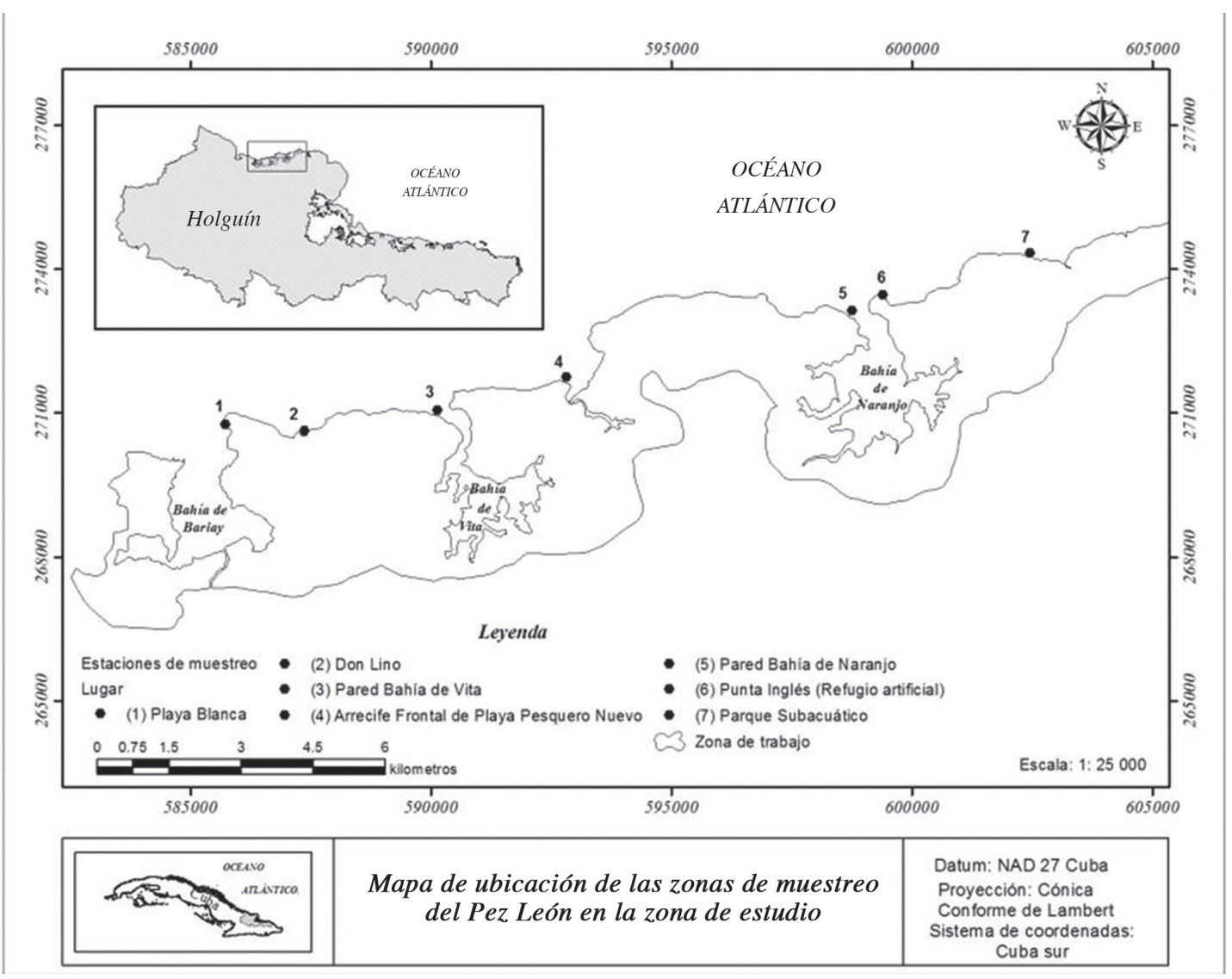

Figura 1. Ubicación geográfica y estaciones de muestreo de la zona de estudio de la provincia de Holguín.

Diseño de muestreo. El estudio se realizó con equipos de buceo Scuba en ocho campañas de muestreo, durante el período comprendido desde abril hasta noviembre de 2016. Se seleccionó un total de siete sitios de monitoreo, distribuidos en dos municipios de la provincia Holguín (Rafael Freyre y Banes). Estos fueron ubicados en diferentes tipos de hábitats, en dependencia de la abundancia y distribución del Pez León en los mismos, priorizando los que podían ofrecer mayor cantidad de refugios (arrecifes coralinos, estructuras artificiales, fondos rocosos y estuarios). En cada sitio de muestreo se tomaron datos que permitieron la descripción de cada tipo de hábitat, como rasgos topográficos y profundidad. Cada sitio fue georreferenciado con sistema de posicionamiento global, GPS Garmin X12 (Fig. 1). 
Método de muestreo. Se utilizó el protocolo nacional elaborado para el estudio del Pez León en Cuba (Chevalier et al., 2008). En cada sitio de muestreo se realizaron seis recorridos lineales de $50 \mathrm{~m}$ de largo por $2 \mathrm{~m}$ de ancho $\left(100 \mathrm{~m}^{2}\right)$, cubriendo un área total de $600 \mathrm{~m}^{2}$. Se contaron todos los individuos observados del Pez León, estimando y anotando la talla de cada ejemplar, además de realizar capturas con fusiles de pesca. Atendiendo a los hábitos conductuales de esta especie, los recorridos lineales se hicieron sobre zonas con el relieve más irregular dentro de cada sitio; buscando con detenimiento en grietas, oquedades y solapas donde pueda refugiarse. Las mediciones de la talla y el peso de los individuos capturados se realizaron con cinta métrica y balanza digital Sartorio.

Análisis de los resultados. La abundancia se calculó en cada estación promediando los valores de cada recorrido lineal (individuo por cada $100 \mathrm{~m}^{2}$ ). Los datos de todas las estaciones fueron comparados con el fin de detectar diferencias entre estaciones y meses del año. Además, se realizó una comparación de la abundancia del Pez León en este año con relación a la abundancia obtenida en años anteriores con el objetivo de conocer si existen diferencias y determinar su comportamiento temporal. Se compararon las tallas y pesos de los individuos capturados entre sitios de muestreo. La comparación múltiple de medias se realizó por PERMANOVA con 10000 permutaciones, empleando la distancia euclidiana, con un nivel de significación del 5\% para una comparación del valor de $p$ sin corrección. Los paquetes estadísticos utilizados fueron el Past. 3.18 (Hammer et al., 2018) y Statistica v.10.0 (StatSoft, 2011).

\section{RESULTADOS}

Distribución y abundancia entre los sitios de muestreo. Se registró un total de 365 individuos del Pez León, en las siete estaciones de monitoreo. El sitio de muestreo con mayor densidad fue Playa Blanca (12.42 ind/100 $\mathrm{m}^{2}$ ), seguido de Pesquero (8.85 ind/100 $\left.\mathrm{m}^{2}\right)$, Bahía de Naranjo $\left(8.83\right.$ ind/100 $\left.\mathrm{m}^{2}\right)$, Bahía de Vita $\left(7.76\right.$ ind $\left./ 100 \mathrm{~m}^{2}\right)$, Parque Subacuático $\left(7.33 \mathrm{ind} / 100 \mathrm{~m}^{2}\right)$, Punta Ingles $\left(6.83 \mathrm{ind} / 100 \mathrm{~m}^{2}\right.$ ) y Don Lino $\left(3.66 \mathrm{ind} / 100 \mathrm{~m}^{2}\right)$, siendo este último el sitio con menor densidad. Se obtuvo diferencia estadística altamente significativa entre Playa Don Lino con Bahía de Naranjo y Bahía de Vita $(p<0.001)$. También se encontró diferencia estadística entre Playa Don Lino con Parque Subacuático, Punta Ingles y Pesquero $(p<0.01)$; y entre Don Lino y Playa Blanca ( $p<0.05$; Fig. 2).

Distribución y abundancia en diferentes meses del año. El mes con mayor densidad durante el 2016 fue abril, con 13.14 ind/100 $\mathrm{m}^{2}$, registrándose diferencias estadísticas significativas en cuanto a la abundancia con el mes de mayo (6.42 ind $\left./ 100 \mathrm{~m}^{2} ; p<0.01\right)$, al igual que julio $\left(7.28 \mathrm{ind} / 100 \mathrm{~m}^{2}\right)$ y agosto $\left(6.71 \mathrm{ind} / 100 \mathrm{~m}^{2}\right)$, con $p<0.05$. También se observó diferencia estadística entre los meses mayo y junio, $(p<0.05$; Fig. 3$)$.

Abundancia entre distintos años. El 2016 fue el año de mayor densidad poblacional del Pez León en la zona costera de uso turístico en la provincia de Holguín, con $8.5 \mathrm{ind} / 100 \mathrm{~m}^{2}$. El segundo año de mayor densidad poblacional fue el $2014\left(7.5 \mathrm{ind} / 100 \mathrm{~m}^{2}\right)$, seguido del 2015 (5.05 ind $\left./ 100 \mathrm{~m}^{2}\right)$, Fig. 4. La comparación de la abundancia poblacional del Pez León registrada en el 2016 en relación a años anteriores demuestra diferencia estadística entre los años 2013 $(p<0.001)$ y $2012(p<0.01)$.

Diferencia de la talla y peso del Pez León entre sitios de muestreo. El sitio con la población de mayor talla fue Playa Blanca $(25.0 \pm 5.3 \mathrm{~cm})$, seguido de Parque Subacuático $(24.8 \pm 4.8 \mathrm{~cm})$, Bahía de Naranjo $(24.7 \pm 5.0 \mathrm{~cm})$, Punta Ingles $(24.6 \pm 5.1 \mathrm{~cm})$, Pesquero $(24.4 \pm 5.2 \mathrm{~cm})$, Don Lino $(24.4 \pm 4.7 \mathrm{~cm})$ y Bahía de Vita $(23.9 \pm 5.6 \mathrm{~cm})$, ver Fig. 5. Se detectó diferencia estadísticas entre Bahía de Naranjo y Don Lino, además de Punta Ingles con Don Lino, Parque Subacuático y Pesquero, respectivamente, para un nivel de significación $p<0.01$. 


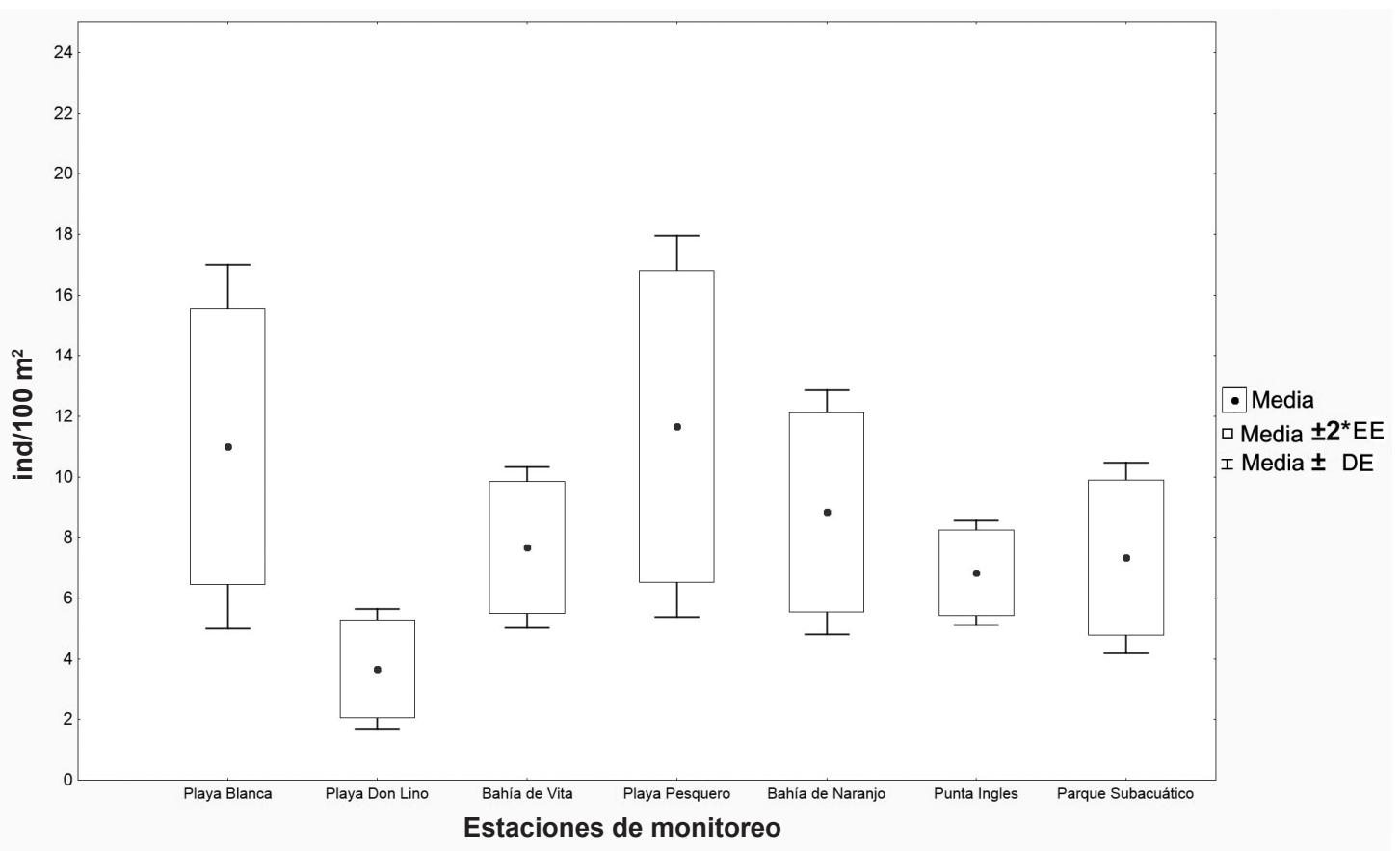

Figura 2. Variación de la abundancia del Pez León entre sitios de muestreo en 2016 a partir de la prueba PERMANOVA con 10000 permutaciones $(\mathrm{P}=0.058$ y $\mathrm{F}=2.2)$. EE: Error estándar; DE: Desviación estándar.

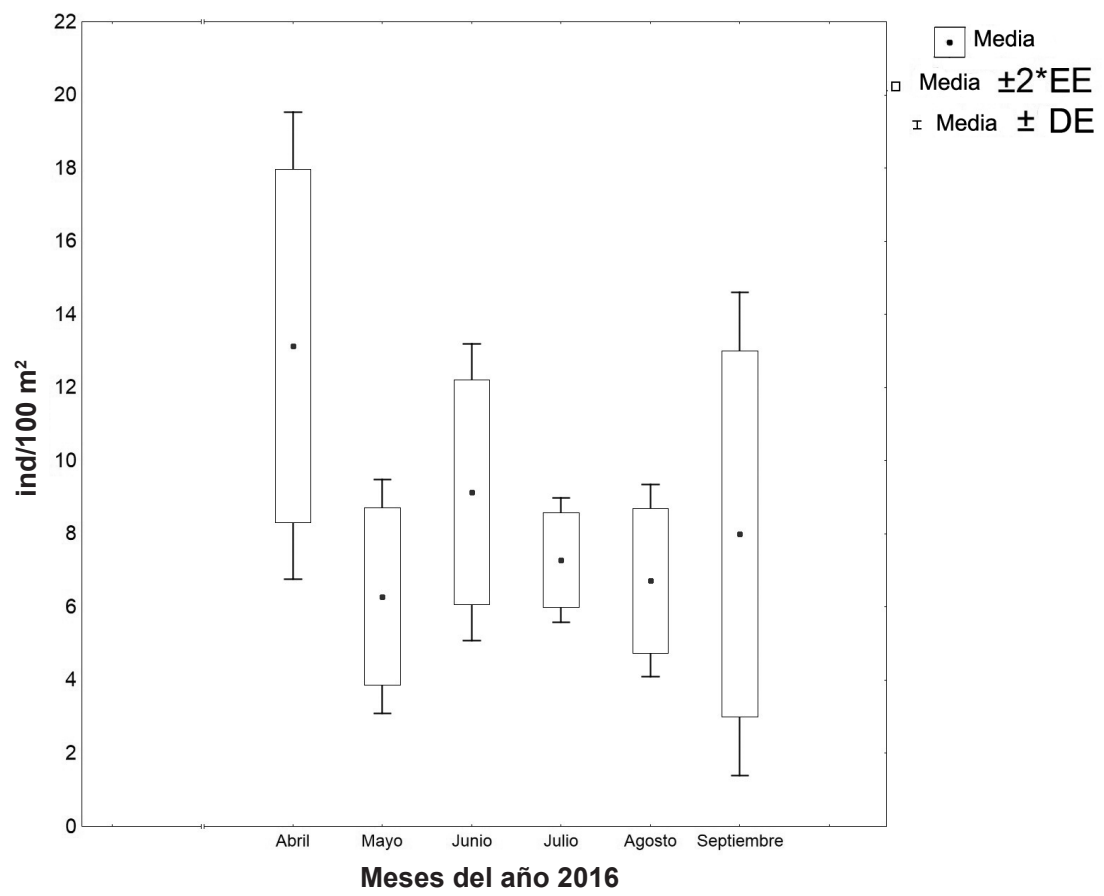

Figura 3. Variación de la abundancia del Pez León entre meses de muestreo en el 2016, a partir de la prueba PERMANOVA con 10000 permutaciones $(\mathrm{P}=0.0473$ y $\mathrm{F}=2.405)$. EE: Error estándar; DE: Desviación estándar. 


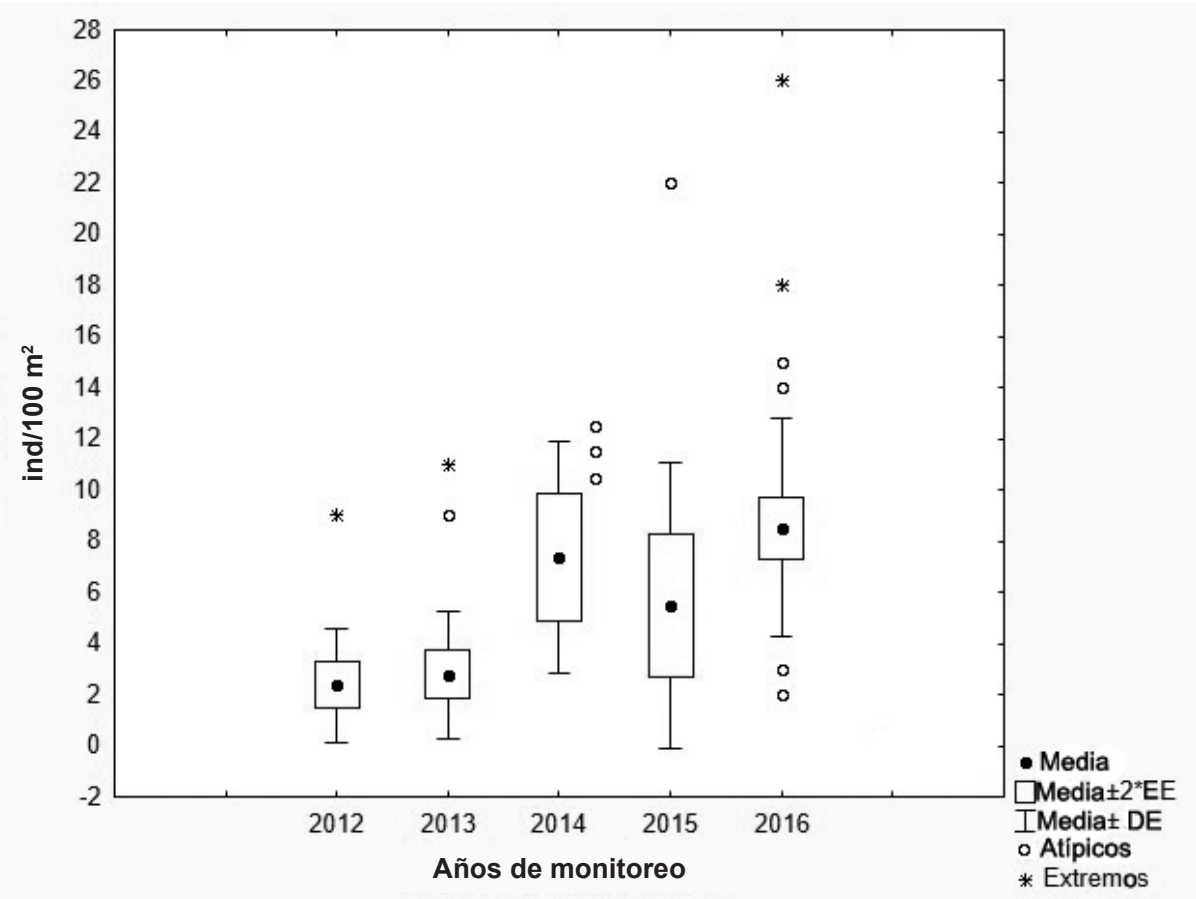

Figura 4. Variación de la abundancia del Pez León entre años a partir de la prueba PERMANOVA con 10000 permutaciones $(\mathrm{P}=0.022$ y $\mathrm{F}=3.653)$. EE: Error estándar; DE: Desviación estándar.

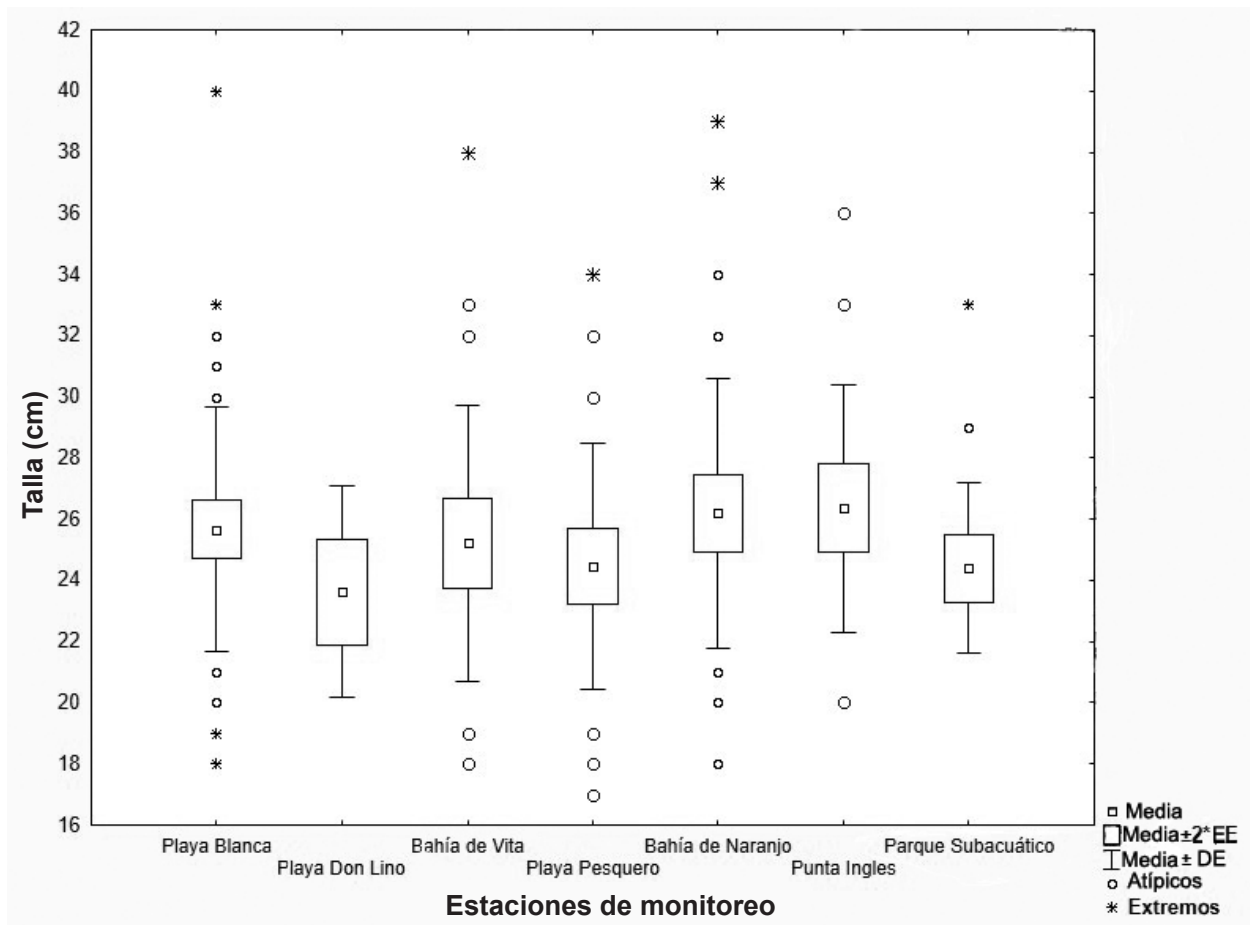

Figura 5. Variación de la talla del Pez León entre sitios de muestreo a partir de la prueba PERMANOVA con 10000 permutaciones $(\mathrm{P}=0.1463$ y $\mathrm{F}=1.562)$. EE: Error estándar; DE: Desviación estándar. 
Con relación al peso, el sitio con la población de mayor biomasa fue Playa Blanca (225.5 \pm 134.9 g), seguido por Parque Subacuático (223.5 \pm 135.7 g), Bahía de Naranjo $(217.8 \pm 139.9 \mathrm{~g})$, Punta Ingles $(214.6 \pm 140.3 \mathrm{~g})$ y Pesquero $(211.4 \pm 139.7 \mathrm{~g})$. Las poblaciones con menor biomasa corporal fueron: Playa Don Lino (209.5 \pm 136.8 g) y Bahía de Vita $(202.9 \pm 143.6 \mathrm{~g})$, ver Fig. 6. Se obtuvieron diferencias muy significativas entre Don Lino con Bahía de Vita y Parque subacuático, respectivamente, además de Bahía de Vita y Pesquero $(p<0.001)$. También se detectó diferencia entre Don Lino con Bahía de Naranjo, Playa Blanca y Punta Ingles, respectivamente; además de Pesquero y Parque Subacuático, Playa Blanca y Bahía de Vita $(p<0.01)$.

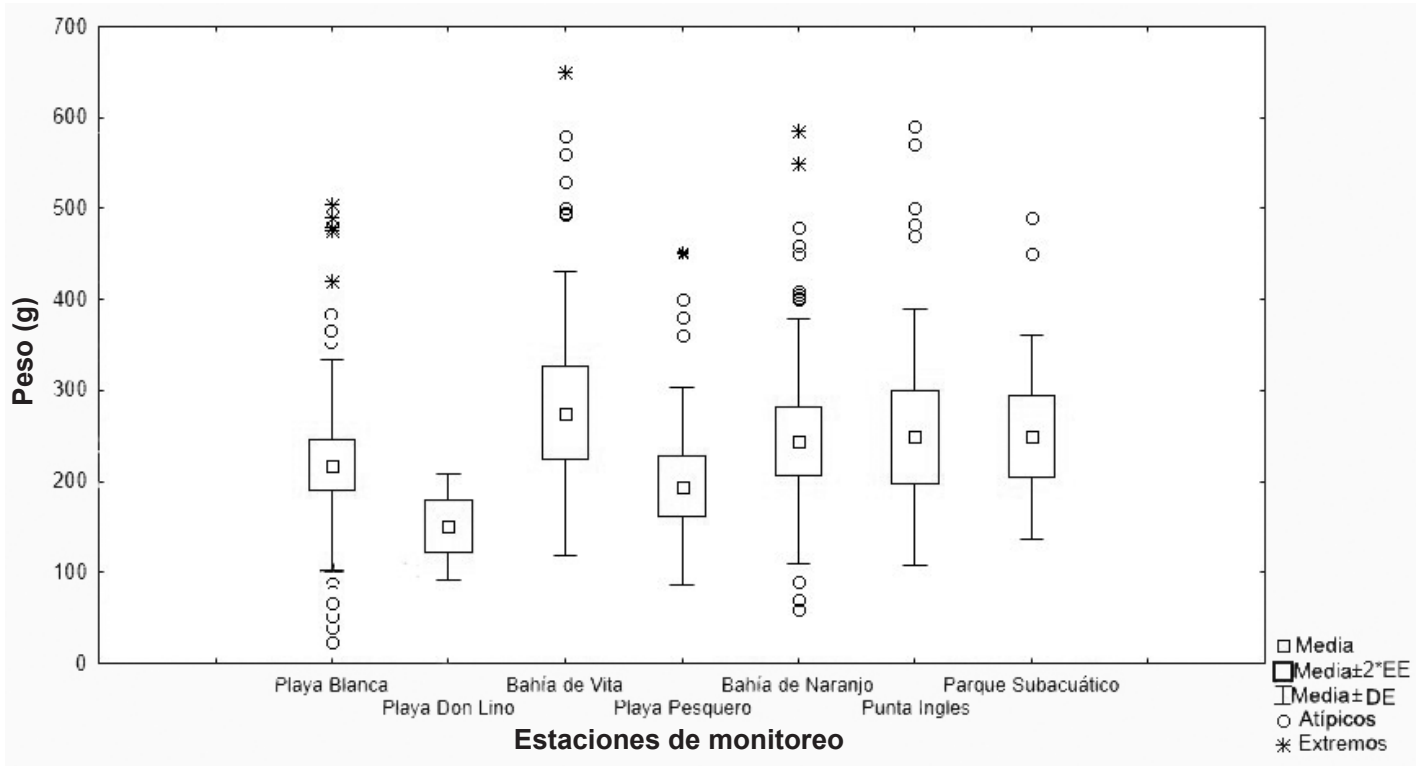

Figura 6. Variación del peso del Pez León entre sitios de muestreo a partir de la prueba PERMANOVA con 10000 permutaciones $(\mathrm{P}=0.0093$ y $\mathrm{F}=2.9)$. EE: Error estándar; DE: Desviación estándar.

\section{DISCUSIÓN}

Distribución y abundancia entre los sitios de muestreo.Analizando el valor total de registros del Pez León, se encuentra que en el año 2016 fue de 365 individuos y en el 2013 de 173 individuos (Vega et al., 2015). Se observa un incremento considerable en el último año de estudio. Esto pudiera estar aconteciendo debido a que el Pez León como especie invasora no tiene depredadores naturales, en la costa norte oriental de Cuba, por lo que se manifiesta una elevada densidad en el tamaño de sus poblaciones, a medida que transcurre el tiempo, además de ocupar un número considerable de biotopos marinos (Cabrera, 2011). También se evidencia que no han sido muy efectivas las acciones llevadas a cabo para el control del Pez León en la zona de uso turístico de Holguín. Por esta razón, se hace necesario incrementar los esfuerzos y disponer de mayores recursos para la eliminación o control de esta especie exótica invasora. Morris y Akins (2009) establecieron los peces como componente principal de la dieta del Pez León y los arrecifes el biotopo con mayor diversidad y abundancia de peces en relación a los manglares y pastizales (Claro, 1994). 
El sitio con la mayor densidad de Pterois volitans fue Playa Blanca $\left(12.42 \mathrm{ind} / 100 \mathrm{~m}^{2}\right)$ y el de menor densidad Don Lino (3.66 ind $/ 100 \mathrm{~m}^{2}$ ). Playa Blanca fue el sitio de mayor dificultad a la hora realizar los censos y capturas, los especímenes se encontraban en cuevas submarinas, lo que dificultaba su visualización. Estas cavernas brindan un refugio seguro contra posibles depredadores, además de ser un sitio con una elevada riqueza de especies ictiológicas, posibilitándole al Pez León una mayor disponibilidad de alimento. Playa Blanca presenta un fondo rocoso con cabezos coralinos y cavernas sumergidas formados sobre una explanada rocosa abrasiva con abundantes algas calcáreas y corales aislados a una profundidad entre 18-20 m (Vega et al., 2015).

Una situación diferente se presenta en Playa Don Lino, un sitio con pocos refugios y disponibilidad de alimento. Este lugar está constituido por un arrecife frontal ubicado en el sector Este. Presenta fondos rocosos coralinos con abundantes macroalgas pardas de la especie Lobophora variegata, cabezos coralinos aislados y profundidad entre 15 y $30 \mathrm{~m}$.

Las diferencias significativas que existen en cuanto a la abundancia entre Playa Don Lino y el resto de los sitios de muestreo durante el período de estudio pueden estar relacionadas con la menor disponibilidad de alimento y refugios naturales, como arrecifes coralinos, fondos duros, fondos no consolidados $\mathrm{y}$ de vegetación sumergida, como pastos $\mathrm{y}$ macroalgas (Vega et al., 2015).

Comparando el mayor valor de densidad obtenido que fue en Playa Blanca $\left(12.42 \mathrm{ind} / 100 \mathrm{~m}^{2}\right)$, con los reportados en los años 2004 y 2008 frente a la costa de Carolina del Norte $\left(0.21 \mathrm{ind} / 100 \mathrm{~m}^{2}, 1.5 \mathrm{ind} / 100 \mathrm{~m}^{2}\right)$, nuestro valor fue 60 y 8.28 veces superior a los antes mencionados (Whitfield et al., 2007; Morris y Whitfield, 2009). En Bahamas (2008), se registraron densidades de Pez León superiores a $3.93 \mathrm{ind} / 100 \mathrm{~m}^{2}$ en tres zonas frente a las costas de New Providence (Green y Côté, 2009), resultando 0.31 veces inferiores al obtenido en Playa Blanca durante nuestro estudio $\left(12.42 \mathrm{ind} / 100 \mathrm{~m}^{2}\right)$. Una evaluación regional de la densidad de Pterois spp. a lo largo del Indopacífico, su región de origen, arrojó una densidad máxima de 0.26 ind $/ 100 \mathrm{~m}^{2}$ (Kulbicki et al., 2012).

Distribución y abundancia en diferentes meses del año. El mes con mayor densidad durante el 2016 fue abril con $13.14 \mathrm{ind} / 100 \mathrm{~m}^{2}$, registrándose diferencias estadísticas significativas en cuanto a la abundancia entre este y los meses de mayo $\left(6.42 \mathrm{ind} / 100 \mathrm{~m}^{2}\right)$, julio $\left(7.28 \mathrm{ind} / 100 \mathrm{~m}^{2}\right)$ y agosto (6.71 ind/100 $\mathrm{m}^{2}$; Fig. 4). Las diferencias entre el mes de abril con los meses de mayo, julio y agosto pudieran estar relacionadas a la incidencia de la extracción sistemática en estos sitios como medida de manejo.

Abundancia entre distintos años. El 2016 fue el año de mayor densidad del Pez León en la zona costera de uso turístico en la provincia de Holguín, con $8.5 \mathrm{ind} / 100 \mathrm{~m}^{2}$ en relación a años anteriores. A este le siguen los años 2014, con $7.5 \mathrm{ind} / 100 \mathrm{~m}^{2}$ y 2015 , con $5.05 \mathrm{ind} / 100 \mathrm{~m}^{2}$ (Fig. 4). La comparación de la abundancia poblacional del Pez León registrada en el 2016, en relación a años anteriores, demuestra diferencia estadística entre los años $2013(p<0.001)$ y $2012(p<0.01)$.

\section{Diferencia de la talla y peso del Pez León entre sitios de muestreo.}

Talla. Los sitios con las poblaciones de mayores tallas como promedio fueron Playa Blanca $(25.0 \pm 5.3 \mathrm{~cm})$, seguido de Parque Subacuático $(24.8 \pm 4.8 \mathrm{~cm})$, Bahía de Naranjo $(24.7 \pm 5.0 \mathrm{~cm})$ y Punta Ingles $(24.6 \pm 5.1 \mathrm{~cm})$. Playa Blanca fue el sitio con individuos de mayor talla, debido probablemente al hecho de tener un mayor número de cabezos coralinos y cuevas submarinas, además de presentar una mayor riqueza de especies ictiológicas. 
Vega y colaboradores (2015) reportaron Playa Pesquero como el sitio de mayor abundancia del Pez León y con los ejemplares de mayor tamaño, capturando individuos con una talla promedio de $27.6 \mathrm{~cm}$ (gama: 16-35 cm). Estos resultados nos permiten inferir cómo las poblaciones del Pez León son capaces de trasladarse a diferentes sitios marinos. Pudieran ser muchas las causas de este comportamiento, como la frecuente pesca submarina en una zona determinada. Los pescadores locales corroboran que en Playa Blanca han observado ejemplares de mayor talla (Vega et al., 2015).

Con relación a la diferencia estadística en la talla del Pez León entre Bahía de Naranjo y Don Lino, además de Punta Ingles con Don Lino, Parque Subacuático y Pesquero, respectivamente, creemos que éstas pudieran estar dadas por las características físicas y condiciones medioambientales de cada localidad. Estos resultados reflejan que Playa Blanca presenta las condiciones más favorables para que el Pez León pueda alcanzar una mayor talla y peso, estas condiciones pueden deberse a una mayor disponibilidad de alimentos y refugios para escapar de los posibles depredadores, a diferencia de Bahía de Vita y Don Lino, sitios que presentaron las poblaciones de menor talla.

Los machos suelen crecer más que las hembras y el mayor individuo de Pez León macho que se ha registrado midió $47.6 \mathrm{~cm}$ LT (J. Morris, comunicación personal), siendo este valor 1.6 veces superior al obtenido en Playa Blanca como el sitio que contiene los individuos de mayor tamaño.

Peso. Los sitios que presentaron los individuos de mayor peso coincidieron con los de mayor talla, posiblemente debido a los mismos factores explicados anteriormente en relación a la talla. El mayor peso registrado en nuestro estudio para un ejemplar $(650 \mathrm{~g})$ es inferior al reportado para el Parque Nacional de Arrecifes Xcalak (PNAX) en Quintana Roo (2011), el cual fue de 859 g. Se hace necesario continuar monitoreando el peso del Pez León en los mismos sitios de captura, debido a que se ha registrado un incremento del peso promedio por año; en el 2009, 2010 y 2011 se obtuvieron valores de $31.1 \mathrm{~g}, 45.6$ g y 120.4 g respectivamente (Sabido et al., 2012). Fishelson (1975) estimó que el peso corporal de individuos adultos de Pterois volitans se encuentra en el rango de 300-500 g, resultando 0.76 veces menor que el valor máximo registrado en nuestro estudio. Sin embargo, se ha reportado un peso máximo de $2000 \mathrm{~g}$ en Bahamas y Colombia (Albins y Lyons, 2012).

En el litoral turístico de Holguín es necesario un control adecuado del Pez León, sobre todo en áreas de reproducción y cría, para evitar el aumento de sus poblaciones y el impacto local que puedan causar a los ecosistemas que prestan bienes y servicios a la industria turística. Es fundamental, además, el control en zonas claves como los sitios de buceo recreativo, los cuales ya se encuentran impactados por más de dos décadas de explotación. El impacto que pueda estar causando el Pez León a la biodiversidad marina de esta región del país se acentúa con el desarrollo de otras actividades antrópicas, como la pesca furtiva submarina, la cual es intensa a pesar de las regulaciones pesqueras de esta zona.

\section{CONCLUSIÓN}

La mayor densidad poblacional de Pez León (12.42 ind/100 $\mathrm{m}^{2}$ ), fue registrada en Playa Blanca, donde se registraron los individuos de mayor talla y peso corporal $(25.0 \mathrm{~cm}) \mathrm{y}$ $(225.5 \mathrm{~g})$ respectivamente. Playa Don Lino fue el sitio de menor densidad $\left(3.66 \mathrm{ind} / 100 \mathrm{~m}^{2}\right)$, Bahía de Vita el sitio con los individuos de menor talla y peso $(23.9 \mathrm{~cm})$ y $(202.9 \mathrm{~g})$, respectivamente. El mes de mayor densidad del Pez León registrado en el año 2016 fue abril (13.14 ind/100 $\mathrm{m}^{2}$ ). 


\section{AGRADECIMIENTOS}

Este trabajo se realizó en el marco de proyecto nacional "Mejorando la prevención, control y manejo de especies exóticas invasoras en ecosistemas vulnerables en Cuba" auspiciado por el GEF/ PNUD y coordinado por el Centro Nacional de Áreas Protegidas (CNAP).

\section{LITERATURA CITADA}

Albins, M. A. y M. A. Hixon. 2008. Invasive Indo-Pacific lionfish (Pterois volitans) reduce recruitment of Atlantic coral-reef fishes. Marine Ecology Progress Series, 367: 233-238.

Albins, M. A. y M. A. Hixon. 2011. Worst case scenario: potential long-term effects of invasive predatory lionfish (Pterois volitans) on Atlantic and Caribbean coral-reef communities. Environmental Biology of Fishes, DOI: 10.1007/s10641-011-9795-1.

Albins, M. A. y P. J. Lyons. 2012. Invasive red lionfish Pterois volitans blow directed jets of water at prey fish. Marine Ecology Progress Series, 448: 1-5.

Alcolado, P. 2008. El pez león amenaza los arrecifes de Cuba. Proyecto PNUD/GEF SabanaCamagüey, 9 pp.

Caballero, A. H., P. P. Chevalier, M. A. Fernando, C. M. Varela, V. Isla y O. Hernández. 2008. Informe de la 1ra expedición piloto del proyecto, estudios preliminares sobre la presencia del pez león (Teleostei: Scorpaenidae: Pterois sp.) en aguas cubanas. Desde Cayo Mono hasta Cayo Guillermo. Acuario Nacional de Cuba, 6 pp.

Cabrera, E. 2011. Abundancia y dieta de Pterois volitans/miles (Teleostei: Scorpaenidae) en varias localidades de Cuba. Tesis de diploma. Centro de investigaciones marinas. Universidad de La Habana, 46 pp.

Chevalier, P., E. Gutiérrez, D. Ibarzabal, S. Romero, V. Isla, J. Calderín y E. Hernández. 2008. Primer registro de Pterois volitans (Pisces:Scorpaenidae) para aguas cubanas. Solenodon, 7: $37-40$.

Claro, R. 1944. Características generales de la ictiofauna. En: Claro, R. (ed.) Ecología de los peces marinos de Cuba. Instituto de Oceanología y CIQRO, México, 55-70 pp.

Fishelson, L. 1975. Ethology and reproduction of pteroid fishes found in the Gulf of Agaba (Red Sea), especially Dendrochirus brachypterus (Cuvier), (Pteroidae, Teleostei). Pubblicazioni della Stazione zoologica di Napoli, 39: 635-656.

Green, S. J. y I. M. Côté. 2009. Record densities of Indo-Pacific lionfish on Bahamian coral reefs. Coral Reefs, 28: 107.

Green, S. J., J. L. Akins y J. A. Morris. 2012. Lionfish dissection: Techniques and applications. NOAA Technical Memorandum NOS NCCOS, 139: 1-24.

Hammer, Ø., D. A. T Harper y P. D. Ryan. 2018. PAST: Paleontological Statistics Software Package for Education and Data Analysis. Palaeontologia Electronica, 4: 1-9. 
Kulbicki, M., J. Beets, P. Chapanet, K. Cure, E. Darling, S. R. Floeter, R. Galzin, A. Green, M. Harmelin-Vivien, M. Hixon, Y. Letourneur, T. Lison de Loma, T. McClanahan, J. McIlwain, G. MouTham, R. Myers, J. K. O’Leary, S. Planes, L. Vigliola y L. Wantiez. 2012. Distribution of Indo-Pacific lionfishes Pterois spp. in their native ranges: implications for the Atlantic invasion. Marine Ecology Progress Series, 446: 189-205.

Morris, J. A. y J. L. Akins. 2009. Feeding ecology of invasive lionfish (Pterois volitans) in the Bahamian archipelago. Environmental Biology of Fishes, 86: 389-398.

Morris, J. A. Jr. y P. E. Whitfield. 2009. Biology, ecology, control and management of the invasive Indo-Pacific lionfish: An updated integrated assessment. NOAA Technical Memorandum NOS NCCOS, 99: 57 pp.

Morris, J. A., K. W. Shertzer y J. A. Rice. 2011. A stage-based matrix population model of invasive lionfish with implications for control. Biological Invasions, 13: 7-12.

Sabido, I. M., P. A. Gómez, Q. A. Medina, R. M. García y L. W. López. 2012. Dinámica Poblacional del Pez León (Pterois volitans) en El Parque Nacional Arrecifes de Xcalak (PNAX), Quintana Roo, Caribe Mexicano. Proceedings of the 64th Gulf and Caribbean Fisheries Institute, 64: 67-64.

StatSoft, Inc. 2011. STATISTICA (data analysis software system), version 10. www.statsoft.com.

Vega, A., E. Reynaldo, A. Fernández, J. Cruz, F. Ocaña y E. Córdova. 2015. Abundancia y distribución del pez león Pterotis volitans (Teleostei: Scorpaenidae) en el litoral turístico de Holguín, Cuba. Solenodon, 12: 72-83. http://www.caribbeanahigroup.org/solenodon12. html.

Whitfield, P. E., J. A. Hare, A. W. David, S. L. Harter, R. C. Muñoz, y C. M. Addison. 2007. Abundance estimates of the Indo-Pacific lionfish Pterois volitans/miles complex in the Western North Atlantic. Biological Invasions, 9: 53-64. 